\title{
Discrete solitons in electromechanical resonators
}

\author{
M. Syafwan 7 H. Susanto, and S. M. Cox \\ School of Mathematical Sciences, University of Nottingham, University Park, Nottingham, NG7 2RD, UK
}

\begin{abstract}
We consider a parametrically driven Klein-Gordon system describing micro- and nano-devices, with integrated electrical and mechanical functionality. Using a multiscale expansion method we reduce the system to a discrete nonlinear Schrödinger equation. Analytical and numerical calculations are performed to determine the existence and stability of fundamental bright and dark discrete solitons admitted by the Klein-Gordon system through the discrete Schrödinger equation. We show that a parametric driving can not only destabilize onsite bright solitons, but also stabilize intersite bright discrete solitons and onsite and intersite dark solitons. Most importantly, we show that there is a range of values of the driving coefficient for which dark solitons are stable, for any value of the coupling constant, i.e. oscillatory instabilities are totally suppressed. Stability windows of all the fundamental solitons are presented and approximations to the onset of instability are derived using perturbation theory, with accompanying numerical results. Numerical integrations of the Klein-Gordon equation are performed, confirming the relevance of our analysis.
\end{abstract}

PACS numbers: $\quad 05.45 .-\mathrm{a}, 63.20 . \mathrm{Pw}, 85.85 .+\mathrm{j}$

\section{INTRODUCTION}

Current advances in the fabrication and control of electromechanical systems on a micro and nanoscale bring many technological promises [1]. These include efficient and highly sensitive sensors to detect stresses, vibrations and forces at the atomic level, to detect chemical signals, and to perform signal processing [2]. As a particular example, a nanoelectromechanical system (NEMS) can detect the mass of a single atom, due to its own very small mass 3,4 , $]$.

On a fundamental level, NEMS with high frequency will allow research on quantum mechanical effects. This is because NEMS, as a miniaturization of microelectromechanical systems (MEMS), can contain a macroscopic number of atoms, yet still require quantum mechanics for their proper description. Thus, NEMS can be considered as a natural playground for a study of mechanical systems at the quantum limit and quantumto-classical transitions (see, e.g., Ref. 5 and references therein).

Typically, nanoelectromechanical devices comprise an electronic device coupled to an extremely high frequency nanoresonator. A large number of arrays of MEMS and NEMS resonators have recently been fabricated experimentally (see, e.g., Ref. [6]). One direction of research in the study of such arrays has focused on intrinsic localized modes (ILMs) or discrete breathers. ILMs can be present due to parametric instabilities in an array of oscillators [7]. ILMs in driven arrays of MEMS have been observed experimentally [8, 9, 10].

Motivated by a recent experiment of Buks and Roukes [6] that succeeded in fabricating and exciting an

* On leave from Department of Mathematics, Faculty of Mathematics and Natural Sciences, Andalas University, Limau Manis, Padang, Indonesia 25163 array of MEMS and measuring oscillations of the resonators, here we consider the equation [11]

$$
\ddot{\varphi}_{n}=D \Delta_{2} \varphi_{n}-\left[1-H \cos \left(2 \omega_{p} t\right)\right] \varphi_{n} \pm \varphi_{n}^{3},
$$

which governs the oscillation amplitude of such an array. Equation (10) is a simplified model of that discussed in Ref. 12, subject to an assumption that the piezoelectric parametric drive is applied directly to each oscillator [13]. The variable $\varphi_{n}$ represents the oscillation amplitude of the $n$th oscillator from its equilibrium position, $D$ is a dc electrostatic nearest-neighbor coupling term, $H$ is a small ac component with frequency $2 \omega_{p}$ responsible for the parametric driving, $\Delta_{2} \varphi_{n}=\varphi_{n+1}-2 \varphi_{n}+\varphi_{n-1}$ is the discrete Laplacian, the dot denotes the derivative with respect to $t$, and the 'plus' and 'minus' signs of the cubic term correspond to a 'softening' and 'stiffening' nonlinearity, respectively. Here, we assume ideal oscillators, so there is no damping present. The creation, stability, and interactions of ILMs in (1) with low damping and in the strong-coupling limit, have been investigated in Ref. 11 . Here, we extend that study to the case of small coupling parameter $D$.

In performing our analysis of the governing equation (11), we introduce a small parameter $\epsilon \ll 1$, and assume that the following scalings hold:

$$
D=\epsilon^{2} 3 C, \quad H=\mp \epsilon^{2} 3 \gamma, \quad \omega_{p}=1 \mp \epsilon^{2} 3 \Lambda / 2 .
$$

We then expand each $\varphi_{n}$ in powers of $\epsilon$, with the leadingorder term being of the form

$$
\varphi_{n} \sim \epsilon\left(\psi_{n}\left(T_{2}, T_{3}, \ldots\right) e^{-i T_{0}}+\bar{\psi}_{n}\left(T_{2}, T_{3}, \ldots\right) e^{i T_{0}}\right),
$$

where $T_{n}=\epsilon^{n} T$. Then the terms at $\mathcal{O}\left(\epsilon^{3} e^{-i T_{0}}\right)$ in (1) yield the following equation for $\psi_{n}$ (see Refs. 14, 15 for a related reduction method):

$$
-2 i \dot{\psi}_{n}=3 C \Delta_{2} \psi_{n} \mp 3 \gamma \bar{\psi}_{n} e^{ \pm i 3 \Lambda T_{2}} \pm 3\left|\psi_{n}\right|^{2} \psi_{n},
$$

where the dot now denotes the derivative with respect to $T_{2}$. Correction terms in Eq. (2) are of order 
$\mathcal{O}\left(\epsilon e^{ \pm i(k+1) T_{0}}, \epsilon^{3} e^{ \pm i(k-1) T_{0}}\right), k \in \mathbb{Z}^{+}$. A justification of this rotating wave type approximation can be obtained in, e.g., Ref. 16.

Writing $\psi_{n}\left(T_{2}\right)=\phi_{n}\left(T_{2}\right) e^{ \pm i 3 \Lambda / 2 T_{2}}$, we find that Eq. (3) becomes

$$
-\frac{2}{3} i \dot{\phi}_{n}=C \Delta_{2} \phi_{n} \mp \Lambda \phi_{n} \mp \gamma \bar{\phi}_{n} \pm\left|\phi_{n}\right|^{2} \phi_{n} .
$$

Then, taking $T_{2}=\frac{2}{3} \hat{T}$, we find that the equation above becomes the parametric driven discrete nonlinear Schrödinger (DNLS) equation

$$
i \dot{\phi}_{n}=-C \Delta_{2} \phi_{n} \pm \Lambda \phi_{n} \pm \gamma \bar{\phi}_{n} \mp\left|\phi_{n}\right|^{2} \phi_{n} ;
$$

here the dot denotes the derivative with respect to $\hat{T}$. The softening and stiffening nonlinearity of (11) correspond, respectively, to the so-called focusing and defocusing nonlinearity in the DNLS (5).

In the absence of parametric driving, i.e., for $\gamma=0$, Eq. (5) is known to admit bright and dark solitons in the system with focusing and defocusing nonlinearity, respectively. Discrete bright solitons in such a system have been discussed before, e.g. in Refs. 17, 18, 19, where it was shown that one-excited-site (onsite) solitons are stable and two-excited-site (intersite) solitons are unstable, for any coupling constant $C$. Undriven discrete dark solitons have also been examined [20, 21, 22, 23, 24]; it is known that intersite dark solitons are always unstable, for any $C$, and onsite solitons are stable only in a small window in $C$. Furthermore, an onsite dark soliton is unstable due to the presence of a quartet of complex eigenvalues, i.e., it suffers oscillatory instability.

The parametrically driven DNLS (5) with a focusing nonlinearity and finite $C$ has been considered briefly in Ref. 25, where it was shown that an onsite bright discrete soliton can be destabilized by parametric driving. Localized excitations of the continuous limit of the parametrically driven DNLS, i.e. (5) with $C \rightarrow \infty$, have been considered by Barashenkov et al. in a different context of applications [26, 27, 28, 29, 30, 31, 32]. The same equation also applies to the study of Bose-Einstein condensates, describing the so-called long bosonic Josephson junctions [33, 34].

In this paper, we consider (10) with either softening or stiffening nonlinearities, which admit bright or dark discrete solitons, respectively. The existence and stability of the fundamental onsite and intersite excitations are discussed through the reduced equation (5). Eq. (5) and a corresponding eigenvalue problem are solved numerically for a range of values of the coupling and driving constants, $C$ and $\gamma$, giving stability windows in the $(C, \gamma)$ plane. Analytical approximations to the boundaries of the numerically obtained stability windows are determined through a perturbation analysis for small $C$. From this analysis, we show, complementing the result of Ref. 25, that parametric driving can stabilize intersite discrete bright solitons. We also show that parametric driving can even stabilize dark solitons, for any coupling constant $C$. These findings, which are obtained from the reduced equation (5), are then confirmed by direct numerical integrations of the original governing equation (1).

The present paper is organized as follows. In Sec. II we present the existence and stability analysis of onsite and intersite bright solitons. Analysis of dark solitons is presented in Sec. III. Confirmation of this analysis, through numerical simulations of the Klein-Gordon system (11), is given in Sec. IV. Finally, we give conclusions in Sec. V.

\section{BRIGHT SOLITONS IN THE FOCUSING DNLS}

In this section we first consider the existence and stability of bright solitons in the focusing DNLS equation. For a static solution of (5) of the form $\phi_{n}=u_{n}$, where $u_{n}$ is real-valued and time-independent, it follows that

$$
-C \Delta_{2} u_{n}-u_{n}^{3}+\Lambda u_{n}+\gamma u_{n}=0 .
$$

Once such discrete solitary-wave solutions of (5) have been found, their linear stability is determined by solving a corresponding eigenvalue problem. To do so, we introduce the linearization ansatz

$$
\phi_{n}=u_{n}+\delta \epsilon_{n}
$$

where $\delta \ll 1$, and substitute this into (5), to yield the following linearized equation at $\mathcal{O}(\delta)$ :

$$
i \dot{\epsilon}_{n}=-C \Delta_{2} \epsilon_{n}-2\left|u_{n}\right|^{2} \epsilon_{n}-u_{n}^{2} \bar{\epsilon}_{n}+\Lambda \epsilon_{n}+\gamma \bar{\epsilon}_{n} .
$$

Writing $\epsilon_{n}(t)=\eta_{n}+i \xi_{n}$, we then obtain from Eq. (7) the eigenvalue problem

$$
\left(\begin{array}{c}
\dot{\eta}_{n} \\
\dot{\xi}_{n}
\end{array}\right)=\mathcal{H}\left(\begin{array}{c}
\eta_{n} \\
\xi_{n}
\end{array}\right),
$$

where

$$
\mathcal{H}=\left(\begin{array}{cc}
0 & \mathcal{L}_{+}(C) \\
-\mathcal{L}_{-}(C) & 0
\end{array}\right)
$$

and the operators $\mathcal{L}_{-}(C)$ and $\mathcal{L}_{+}(C)$ are defined by

$$
\begin{aligned}
& \mathcal{L}_{-}(C) \equiv-C \Delta_{2}-\left(3 u_{n}^{2}-\Lambda-\gamma\right), \\
& \mathcal{L}_{+}(C) \equiv-C \Delta_{2}-\left(u_{n}^{2}-\Lambda+\gamma\right)
\end{aligned}
$$

The stability of the solution $u_{n}$ is then determined by the eigenvalues of (8). If we denote these eigenvalues by $i \omega$, then the solution $u_{n}$ is stable only when $\operatorname{Im}(\omega)=0$ for all eigenvalues $\omega$.

We note that, because (8) is linear, we may eliminate one of the eigenvectors, for instance $\xi_{n}$, to obtain an alternative expression of the eigenvalue problem in the form

$$
\mathcal{L}_{+}(C) \mathcal{L}_{-}(C) \eta_{n}=\omega^{2} \eta_{n} \equiv \Omega \eta_{n} .
$$

In view of the relation $\Omega=\omega^{2}$, it follows that a soliton is unstable if it has an eigenvalue with either $\Omega<0$ or $\operatorname{Im}(\Omega) \neq 0$. 


\section{A. Analytical calculations}

Analytical calculations of the existence and stability of discrete solitons can be carried out for small coupling constant $C$, using a perturbation analysis. This analysis exploits the exact solutions of (6) in the uncoupled limit $C=0$, which we denote by $u_{n}=u_{n}^{(0)}$, in which each $u_{n}^{(0)}$ must take one of the three values given by

$$
0, \pm \sqrt{\Lambda+\gamma}
$$

Solutions of (6) for small $C$ can then be calculated analytically by writing

$$
u_{n}=u_{n}^{(0)}+C u_{n}^{(1)}+C^{2} u_{n}^{(2)}+\cdots .
$$

In studying the stability problem, it is natural to also expand the eigenvector $\eta_{n}$ and the eigenvalue $\Omega$ in powers of $C$, as

$\eta_{n}=\eta_{n}^{(0)}+C \eta_{n}^{(1)}+\mathcal{O}\left(C^{2}\right), \quad \Omega=\Omega^{(0)}+C \Omega^{(1)}+\mathcal{O}\left(C^{2}\right)$.

Upon substituting this expansion into Eq. (9) and identifying coefficients of successive powers of the small parameter $C$, we obtain from the equations at $\mathcal{O}(1)$ and $\mathcal{O}(C)$ the results

$$
\begin{aligned}
& {\left[\mathcal{L}_{+}(0) \mathcal{L}_{-}(0)-\Omega^{(0)}\right] \eta_{n}^{(0)}=0} \\
& {\left[\mathcal{L}_{+}(0) \mathcal{L}_{-}(0)-\Omega^{(0)}\right] \eta_{n}^{(1)}=f_{n} \eta_{n}^{(0)},}
\end{aligned}
$$

where

$f_{n}=-\left(\Delta_{2}+2 u_{n}^{(0)} u_{n}^{(1)}\right) \mathcal{L}_{-}(0)-\mathcal{L}_{+}(0)\left(\Delta_{2}+6 u_{n}^{(0)} u_{n}^{(1)}\right)+\Omega^{(1)}$.

In the uncoupled limit, $C=0$, the eigenvalue problem is thus simplified to

$$
\Omega^{(0)}=\mathcal{L}_{+}(0) \mathcal{L}_{-}(0),
$$

from which we conclude that there are two possible eigenvalues, given by

$$
\Omega_{C}^{(0)}=\Lambda^{2}-\gamma^{2}, \quad \Omega_{E}^{(0)}=4(\Lambda+\gamma) \gamma
$$

which correspond, respectively, to the solutions $u_{n}^{(0)}=0$ (for all $n$ ) and $u_{n}^{(0)}= \pm \sqrt{\Lambda+\gamma}$ (for all $n$ ).

We begin by considering bright soliton solutions, for which $u_{n} \rightarrow 0$ as $n \rightarrow \pm \infty$. This then implies that (for $C=0$ ) the eigenvalue $\Omega_{C}^{(0)}$ has infinite multiplicity; it generates a corresponding continuous spectrum (phonon band) for finite positive $C$. To investigate the significance of this continuous spectrum, we introduce a plane wave expansion

$$
\eta_{n}=a e^{i \kappa n}+b e^{-i \kappa n}
$$

from which one obtains the dispersion relation

$$
\Omega=(2 C(\cos \kappa-1)-\Lambda)^{2}-\gamma^{2} .
$$
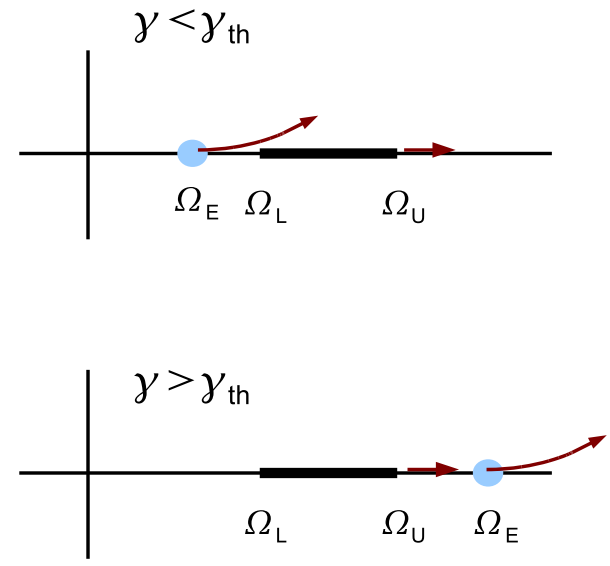

FIG. 1: A sketch of the dynamics of the eigenvalues and the continuous spectrum of a stable onsite bright soliton in the $(\operatorname{Re}(\Omega), \operatorname{Im}(\Omega))$ plane. The arrows indicate the direction of movement as the coupling constant $C$ increases. Note that a soliton is unstable if there is some $\Omega$ with either $\Omega<0$ or $\operatorname{Im}(\Omega) \neq 0$.

This in turn shows that the continuous band lies between

$$
\Omega_{L}=\Lambda^{2}-\gamma^{2}, \text { when } \kappa=0
$$

and

$$
\Omega_{U}=\Lambda^{2}-\gamma^{2}+8 C(\Lambda+2 C), \text { when } \kappa=\pi .
$$

From the continuous spectrum analysis above, it can be concluded that an instability can only be caused by the dynamics of discrete spectrum.

\section{Onsite bright solitons}

The existence and stability of a single excited state, i.e. an onsite bright soliton, in the presence of a parametric driving has been considered in Ref. 25. For small $C$, the soliton is given by 25 .

$$
u_{n}= \begin{cases}\sqrt{\Lambda+\gamma}+C / \sqrt{\Lambda+\gamma}+\mathcal{O}\left(C^{2}\right), & n=0 \\ C / \sqrt{\Lambda+\gamma}+\mathcal{O}\left(C^{2}\right), & n=-1,1 \\ \mathcal{O}\left(C^{2}\right), & \text { otherwise }\end{cases}
$$

and its eigenvalue by

$$
\Omega_{E}=4(\Lambda+\gamma) \gamma+8 \gamma C+\mathcal{O}\left(C^{2}\right)
$$

It was shown in Ref. [25] that the configuration (18), which is known to be stable for any value of $C$ when $\gamma=0$, can be destabilized by parametric driving. Furthermore, it was shown that there are two mechanisms of destabilization, as sketched in Fig. 1. The two instability scenarios are determined by the relative positions of $\Omega_{E}^{(0)}$ and $\Omega_{C}^{(0)}$, as we now summarize. First, we note that there is a threshold value, $\gamma_{\mathrm{th}}=\Lambda / 5$, at which the two leading-order eigenvalues coincide, so that $\Omega_{E}^{(0)}=\Omega_{C}^{(0)}$. 
For $\gamma>\gamma_{\mathrm{th}}$, upon increasing $C$ from $C=0$, the instability is caused by the collision of $\Omega_{E}$ with $\Omega_{U}$; taking $\Omega_{E}=\Omega_{U}$ then yields the corresponding approximate critical value

$$
\gamma_{\mathrm{cr}}^{1}=-\frac{2}{5} \Lambda-\frac{4}{5} C+\frac{1}{5} \sqrt{9 \Lambda^{2}+56 C \Lambda+96 C^{2}}
$$

For $\gamma<\gamma_{\text {th }}$, by contrast, the instability is caused by the collision of $\Omega_{E}$ with an eigenvalue bifurcating from $\Omega_{L}$. In this case, the critical value of $\gamma$ can be approximated by taking $\Omega_{E}=\Omega_{L}$, giving

$$
\gamma_{\mathrm{cr}}^{2}=-\frac{2}{5} \Lambda-\frac{4}{5} C+\frac{1}{5} \sqrt{9 \Lambda^{2}+16 C(\Lambda+C)} .
$$

Together, $\gamma_{\mathrm{cr}}^{1}$ and $\gamma_{\mathrm{cr}}^{2}$ give approximate boundaries of the instability region in the $(C, \gamma)$-plane.
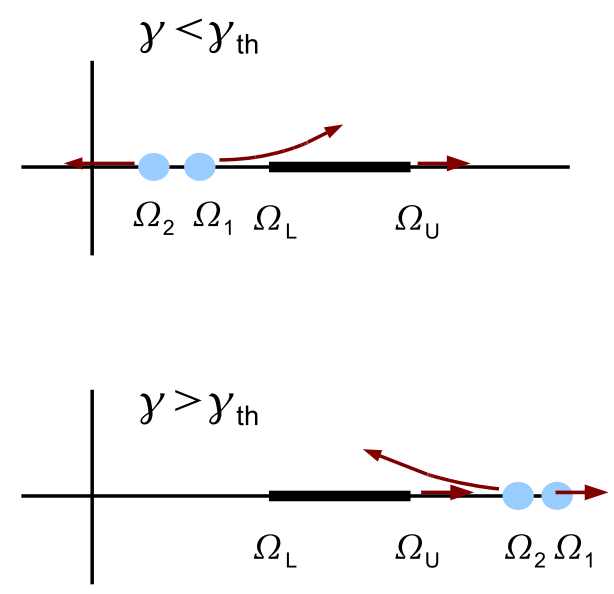

FIG. 2: As Fig. 1, but for a stable intersite bright soliton.

\section{Intersite bright solitons}

The next natural fundamental solution to be considered is an intersite bright soliton, i.e., a two-excitedsite discrete mode. In the uncoupled limit, the mode structure is of the form $u_{n}^{(0)}=0$ for $n \neq 0,1$ and $u_{0}^{(0)}=u_{1}^{(0)}=\sqrt{\Lambda+\gamma}$. Using a perturbative expansion, one can show further that the soliton is given by

$$
u_{n}= \begin{cases}\sqrt{\Lambda+\gamma}+\frac{1}{2} C / \sqrt{\Lambda+\gamma}+\mathcal{O}\left(C^{2}\right), & n=0,1, \\ C / \sqrt{\Lambda+\gamma}+\mathcal{O}\left(C^{2}\right), & n=-1,2, \\ \mathcal{O}\left(C^{2}\right), & \text { otherwise. }\end{cases}
$$

To study the stability of the intersite bright soliton above, let us consider the $\mathcal{O}(1)$ equation (11). Due to the presence of two non-zero excited sites at $C=0$, the soliton (22) has at leading order the double eigenvalue $\Omega_{E}^{(0)}=4(\Lambda+\gamma) \gamma$, with corresponding eigenvectors $\eta_{n}^{(0)}=$ 0 for $n \neq 0,1, \eta_{0}^{(0)}=1$, and $\eta_{1}^{(0)}= \pm 1$.

The continuation of the eigenvalue $\Omega_{E}^{(0)}$ above for nonzero coupling $C$ can be obtained from Eq. (12) by applying a solvability condition. The Fredholm alternative requires that $f_{n}=0$ for all $n$, from which we immediately deduce that the double eigenvalue splits into two distinct eigenvalues, which are given as functions of $C$ by

$$
\Omega_{1}=4(\Lambda+\gamma) \gamma+4 \gamma C+\mathcal{O}\left(C^{2}\right)
$$

and

$$
\Omega_{2}=4(\Lambda+\gamma) \gamma-4(\Lambda+\gamma) C+\mathcal{O}\left(C^{2}\right)
$$

As is the case for onsite discrete solitons, intersite bright solitons can also become unstable. The mechanism of the instability is again determined by the relative positions of $\Omega_{E}^{(0)}$ and $\Omega_{C}^{(0)}$, as sketched in Fig. 2. Performing an analysis corresponding to that in Ref. [25], we find that the two mechanisms of destabilization for an onsite discrete soliton also occur here. The two scenarios have corresponding critical values of $\gamma$, which are given as functions of $C$ by

$$
\begin{gathered}
\gamma_{\mathrm{cr}}^{1}=-\frac{2}{5} \Lambda+\frac{2}{5} C+\frac{1}{5} \sqrt{9 \Lambda^{2}+52 \Lambda C+84 C^{2}} \\
\gamma_{\mathrm{cr}}^{2}=-\frac{2}{5} \Lambda-\frac{2}{5} C+\frac{1}{5} \sqrt{9 \Lambda^{2}+8 \Lambda C+4 C^{2}} .
\end{gathered}
$$

We emphasize, as is apparent from the sketch shown in Fig. 2, that there is another possible mechanism of destabilization for $\gamma<\gamma_{\text {th }}$, namely when $\Omega_{2}$ becomes negative. The third critical choice of parameter values is then obtained by setting $\Omega_{2}=0$, i.e.

$$
\gamma_{\mathrm{cr}}^{3}=C
$$

\section{B. Comparisons with numerical calculations}

We have solved the steady-state equation (6) numerically using a Newton-Raphson method, and analyzed the stability of the numerical solution by solving the eigenvalue problem (8). In this section, we compare these numerical results with the analytical calculations of the previous section. For the sake of simplicity, we set $\Lambda=1$ in all the illustrative examples.

\section{Onsite bright solitons}

Comparisons between numerical calculations and analytical approximations for the case of onsite bright solitons have been fully presented and discussed in Ref. [25]. For the sake of completeness, we reproduce the results of Ref. 25] for the (in)stability domain of onsite solitons in the $(C, \gamma)$ plane in Fig. 3. Approximations (20) and (21) are also shown there.

\section{Intersite bright solitons}

For the stability of intersite bright solitons, we start by examining the validity of our analytical prediction for 


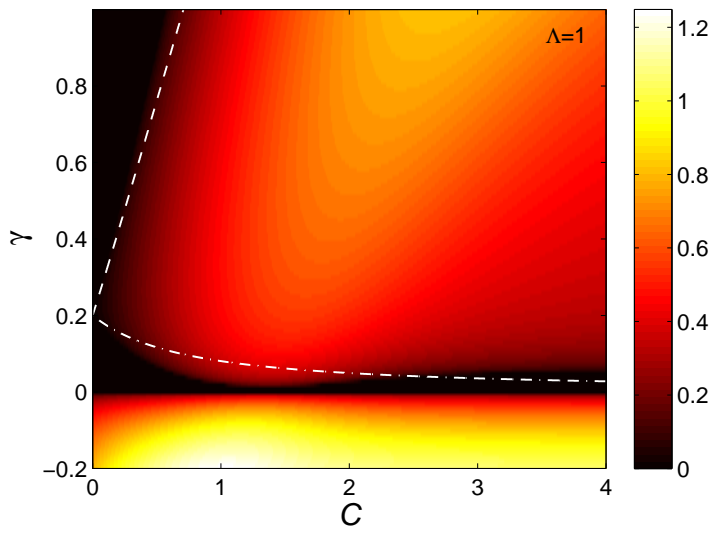

FIG. 3: (Colour online) The (in)stability region of onsite bright solitons in $(C, \gamma)$ space. For each value of $C$ and $\gamma$, the corresponding colour indicates the maximum value of $|\operatorname{Im}(\omega)|$ (over all eigenvalues $\omega$ ) for the steady-state solution at that point. Stability is therefore indicated by the region in which $\operatorname{Im}(\omega)=0$, namely the dark region. White dashed and dashdotted lines give the analytical approximations (20) and (21), respectively.

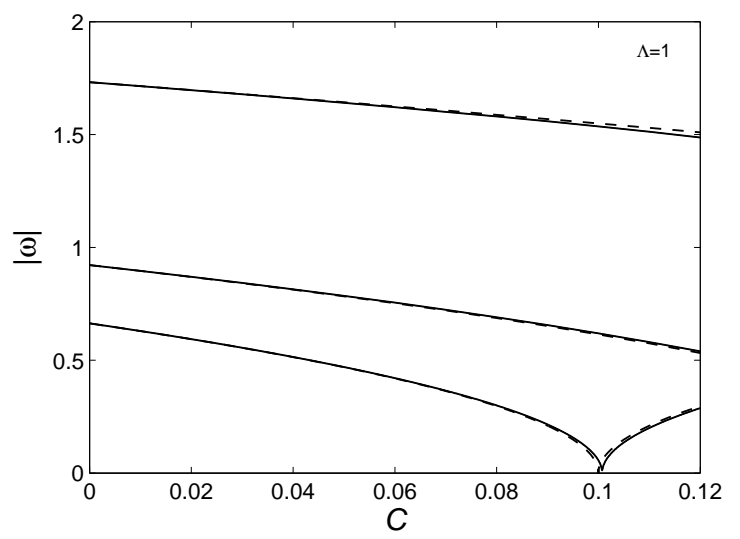

FIG. 4: Comparison between the critical eigenvalue of intersite bright solitons obtained numerically (solid lines) and its analytical approximation (dashed lines). The upper and lower curves correspond, respectively, to $\gamma=0.5$ and $\gamma=0.1$, approximated by Eq. (24), whereas the middle one corresponds to $\gamma=0.18$, approximated by Eq. (23).

the eigenvalue associated with the phase mode as given by Eqs. (23) and (24). In Fig. 4, we present a comparison between the analytical approximation and the numerics for some representative values of $\gamma$ (specifically $\gamma=0.1,0.18,0.5)$. This figure reveals the relative accuracy of the small- $C$ approximations, and we conclude that their range of validity is wider for smaller values of $\gamma$.

Next we turn to a description of the eigenvalue structure of this intersite configuration for the three values of $\gamma$ given above; this is shown in Fig. 5. where the left and right panels respectively present the structure just before and just after the first collision that results in the mode instability. We now describe results in more detail for the three values of $\gamma$ in turn.

For $\gamma=0.1$, when $C=0$ the eigenvalues $\omega$ lie in the gap between the two parts of the continuous spectrum, and the instability is caused by a collision between the critical eigenvalue and its twin at the origin (see the top panels of Fig. 51). For $\gamma=0.18$, the eigenvalues $\omega$ also lie in the gap between the two parts of the continuous spectrum, but the instability in this case is due to a collision between one of the eigenvalues and the inner edge of the continuous spectrum at $\omega= \pm \sqrt{\Omega_{L}}$ (see the middle panels of Fig. (5). In contrast to the two cases above, for $\gamma=0.5$ the eigenvalues lie beyond the continuous spectrum, and the instability is caused by a collision between the critical eigenvalue and the outer boundary at $\omega= \pm \sqrt{\Omega_{U}}$ (see the bottom panels of Fig. 5). All the numerical results presented here are in accordance with the sketch shown in Fig. 2

Numerical calculations of the stability of intersite bright solitons, for a relatively large range of $C$ and $\gamma$, give us the stability domain of the bright solitons in the two-parameter $(C, \gamma)$ plane, which is presented in Fig. 6. We use colours to represent the maximum of $|\operatorname{Im}(\omega)|$ as a function of $C$ and $\gamma$; thus solitons are stable in the black region. Our analytical predictions for the occurrence of instability, given by Eqs. (25)-(27), are also shown, respectively, by dashed, dotted, and dash-dotted lines.

\section{DARK SOLITONS IN THE DEFOCUSING DNLS}

In this section we consider the existence and stability of onsite and intersite dark solitons for the defocusing DNLS equation. Then a static (real-valued, time-independent) solution $u_{n}$ of (5) satisfies

$$
-C \Delta_{2} u_{n}+u_{n}^{3}-\Lambda u_{n}-\gamma u_{n}=0 .
$$

In contrast to bright solitons, where $u_{n} \rightarrow 0$ as $n \rightarrow \pm \infty$, dark solitons have $u_{n} \rightarrow \pm \sqrt{\Lambda+\gamma}$ as $n \rightarrow \pm \infty$.

To examine the stability of $u_{n}$, we again introduce the linearization ansatz $\phi_{n}=u_{n}+\delta \epsilon_{n}$, where again $\delta \ll 1$. Substituting this ansatz into the defocusing equation (5), writing $\epsilon_{n}(t)=\eta_{n}+i \xi_{n}$, and linearizing in $\delta$, we again find

$$
\left(\begin{array}{c}
\dot{\eta}_{n} \\
\dot{\xi}_{n}
\end{array}\right)=\left(\begin{array}{cc}
0 & \mathcal{L}_{+} \\
-\mathcal{L}_{-} & 0
\end{array}\right)\left(\begin{array}{l}
\eta_{n} \\
\xi_{n}
\end{array}\right)=\mathcal{H}\left(\begin{array}{l}
\eta_{n} \\
\xi_{n}
\end{array}\right)
$$

but where the operators $\mathcal{L}_{ \pm}(C)$ are now defined as

$$
\begin{aligned}
& \mathcal{L}_{-}(C) \equiv-C \Delta_{2}+\left(3 u_{n}^{2}-\Lambda-\gamma\right), \\
& \mathcal{L}_{+}(C) \equiv-C \Delta_{2}+\left(u_{n}^{2}-\Lambda+\gamma\right) .
\end{aligned}
$$

The eigenvalue problem above can be simplified further as for the focusing case, to the alternative form

$$
\mathcal{L}_{+}(C) \mathcal{L}_{-}(C) \eta_{n}=\omega^{2} \eta_{n}=\Omega \eta_{n} .
$$




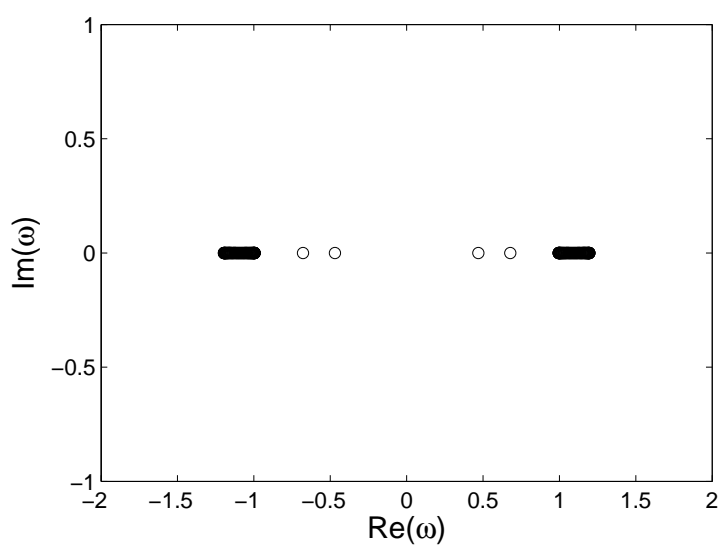

(a) $\gamma=0.1, C=0.05$

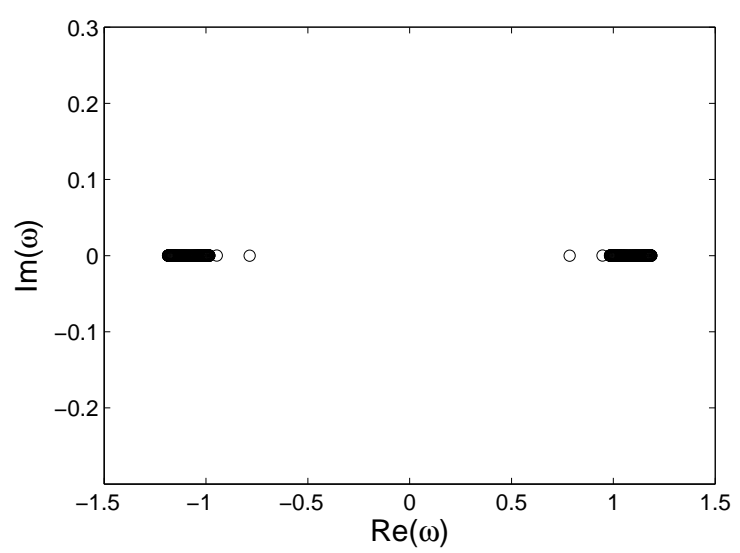

(c) $\gamma=0.18, C=0.05$

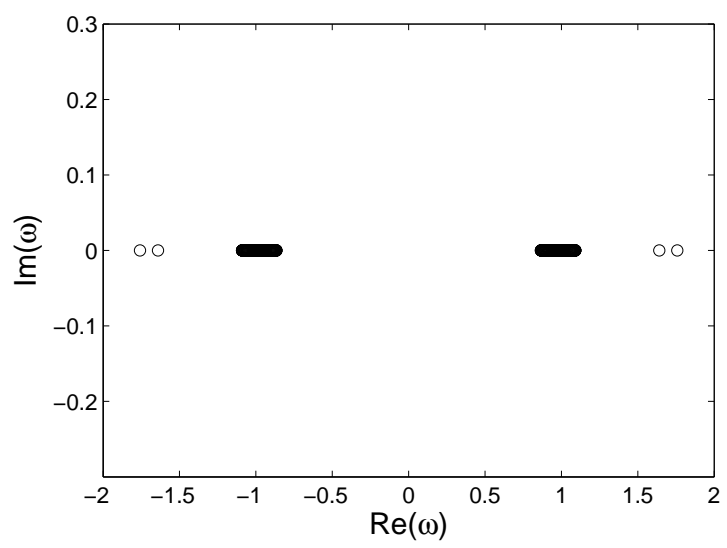

(e) $\gamma=0.5, C=0.05$

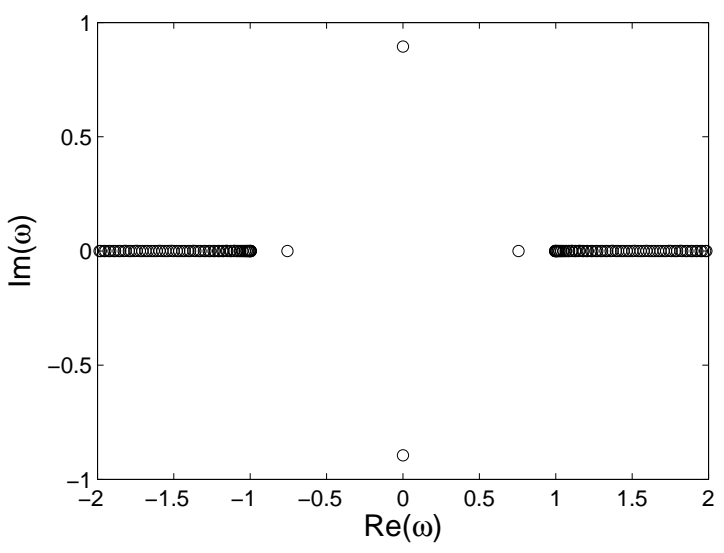

(b) $\gamma=0.1, C=0.3$

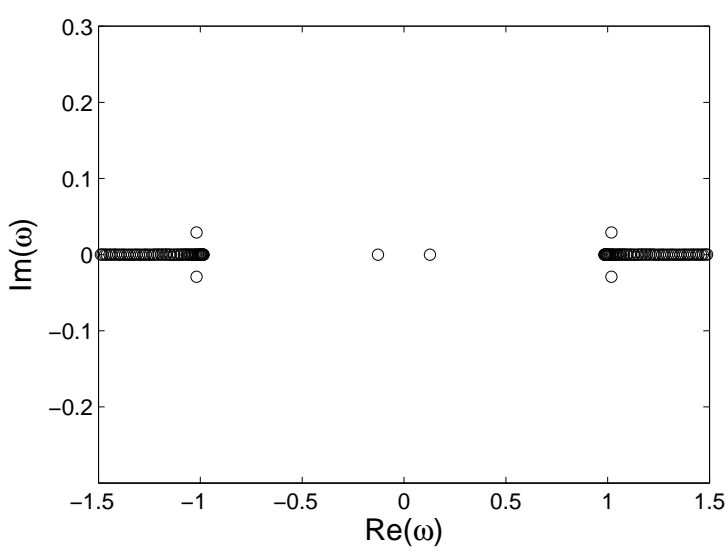

(d) $\gamma=0.18, C=0.18$

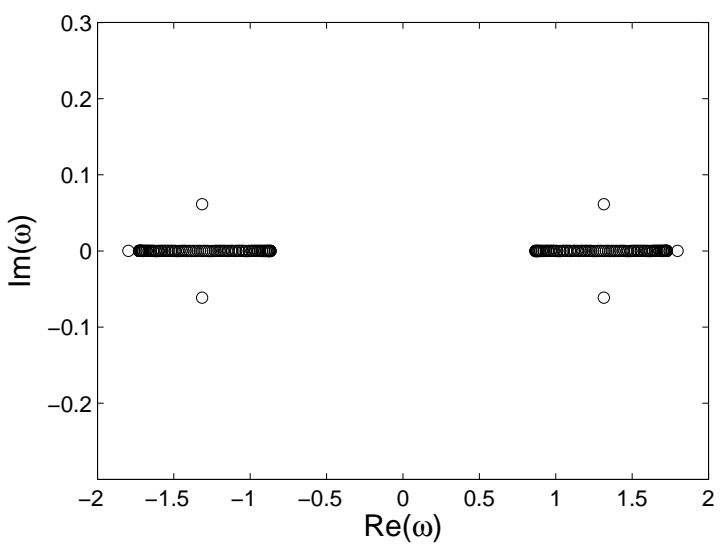

(f) $\gamma=0.5, C=0.2$

FIG. 5: The structure of the eigenvalues of intersite bright solitons in the complex plane for three values of $\gamma$, as indicated in the caption of each plot. Left and right panels depict the eigenvalues of stable and unstable solitons, respectively.

Performing a stability analysis as before, we find the dispersion relation for a dark soliton to be

$$
\Omega=(2 C(\cos \kappa-1)-(\Lambda+2 \gamma))^{2}-\Lambda^{2}
$$

and so the continuous band lies between

$$
\Omega_{L}=4(\Lambda+\gamma) \gamma, \text { when } \kappa=0,
$$

and

$$
\Omega_{U}=4(\Lambda+\gamma) \gamma+8 C(\Lambda+2 \gamma+2 C), \text { when } \kappa=\pi .
$$




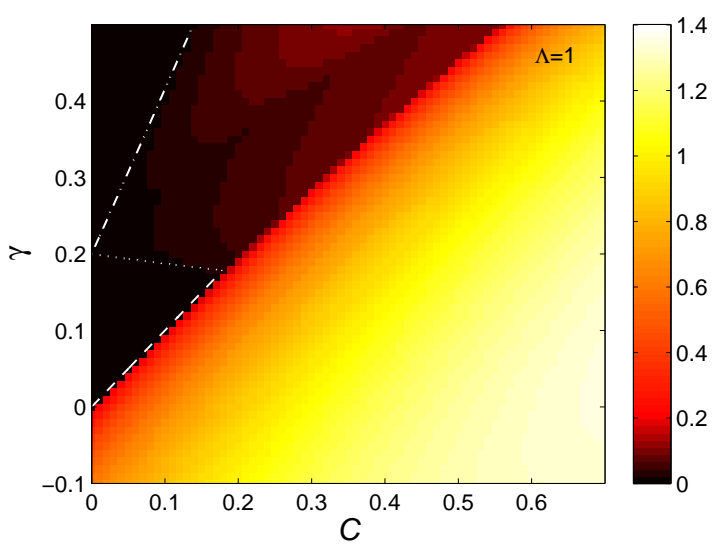

FIG. 6: (Colour online) As Fig. 3 but for intersite bright solitons. Our analytical approximations, given by Eqs. (25), (26) and (27), are shown as white dash-dotted, dotted, and dashed lines, respectively.

\section{A. Analytical calculations}

To study the eigenvalue(s) of the dark soliton analytically, we again expand $\eta_{n}$ and $\Omega$ in powers of $C$, and hence obtain from (30), at $\mathcal{O}\left(C^{0}\right)$ and $\mathcal{O}\left(C^{1}\right)$, respectively, the equations

$$
\left[\mathcal{L}_{+}(0) \mathcal{L}_{-}(0)-\Omega^{(0)}\right] \eta_{n}^{(0)}=0
$$

and

$$
\left[\mathcal{L}_{+}(0) \mathcal{L}_{-}(0)-\Omega^{(0)}\right] \eta_{n}^{(1)}=f_{n}
$$

with

$$
f_{n}=\left(Q_{n}+\Omega^{(1)}\right) \eta_{n}^{(0)}
$$

where

$$
Q_{n}=\left(\Delta_{2}-2 u_{n}^{(0)} u_{n}^{(1)}\right) \mathcal{L}_{-}(0)+\mathcal{L}_{+}(0)\left(\Delta_{2}-6 u_{n}^{(0)} u_{n}^{(1)}\right) .
$$

We next investigate the eigenvalues of both intersite and onsite modes.

\section{Onsite dark solitons}

With errors of order $C^{2}$, an onsite dark soliton is given by

$$
u_{n}= \begin{cases}-\sqrt{\Lambda+\gamma}, & n=-2,-3, \ldots, \\ -\sqrt{\Lambda+\gamma}+\frac{1}{2} C / \sqrt{\Lambda+\gamma}, & n=-1, \\ 0, & n=0, \\ \sqrt{\Lambda+\gamma}-\frac{1}{2} C / \sqrt{\Lambda+\gamma}, & n=1, \\ \sqrt{\Lambda+\gamma}, & n=2,3, \ldots\end{cases}
$$

For this configuration,

$$
\mathcal{L}_{+}(0) \mathcal{L}_{-}(0)= \begin{cases}\Lambda^{2}-\gamma^{2}, & n=0 \\ 4(\Lambda+\gamma) \gamma, & n \neq 0\end{cases}
$$

From Eq. (34), we then deduce that at $C=0$ the eigenvalues of onsite discrete dark solitons are given by $\Omega_{C}^{(0)}=4(\Lambda+\gamma) \gamma$, which becomes the continuous band for nonzero $C$, and $\Omega_{E}^{(0)}=\Lambda^{2}-\gamma^{2}$.

The continuation of the eigenvalue $\Omega_{E}^{(0)}$ for nonzero $C$ can be calculated from Eq. (35). The coefficient of $\eta_{n}^{(1)}$ in this case is given by

$$
\mathcal{L}_{+}(0) \mathcal{L}_{-}(0)-\Omega^{(0)}= \begin{cases}0, & n=0 \\ 4 \Lambda \gamma-\Lambda^{2}+5 \gamma^{2}, & n \neq 0\end{cases}
$$

The solvability condition for (35) then requires that $f_{0}=$ $\left(4 \Lambda-\Omega^{(1)}\right) \eta_{0}^{(0)}=0$. Setting $\eta_{0}^{(0)} \neq 0$, we deduce that $\Omega^{(1)}=-4 \Lambda$. Hence the eigenvalue of an onsite dark soliton for small $C$ is given by

$$
\Omega=\Lambda^{2}-\gamma^{2}-4 \Lambda C+\mathcal{O}\left(C^{2}\right) .
$$

Initially, i.e. for $C=0$, the relative positions of the eigenvalue and the continuous spectrum can be divided into two cases, according to whether $\gamma \gtrless \gamma_{\text {th }}=\Lambda / 5$. When $C=0$ and $\gamma<\gamma_{\text {th }}\left(\gamma>\gamma_{\text {th }}\right)$ the eigenvalue (41) will be above (below) the continuous spectrum, as sketched in Fig. 7. These relative positions determine the instability mechanism for an onsite dark soliton, as we now describe.
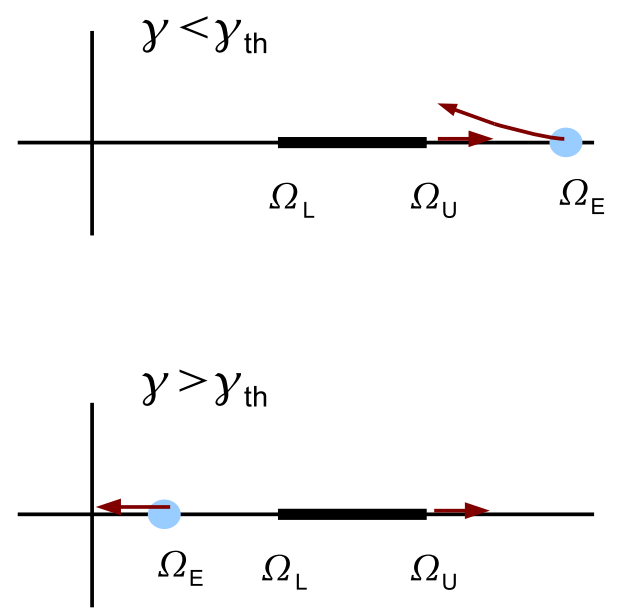

FIG. 7: As Fig. 1, but for a stable onsite dark soliton.

For $\gamma<\Lambda / 5$, the instability is due to a collision between the eigenvalue (41) and $\Omega_{U}$, which approximately occurs when $\gamma=\gamma_{\mathrm{cr}}^{1}$, where

$$
\gamma_{\mathrm{cr}}^{1}=-\frac{2}{5} \Lambda-\frac{8}{5} C+\frac{1}{5} \sqrt{9 \Lambda^{2}-28 \Lambda C-16 C^{2}}
$$

note that this critical value is meaningful only when $C \leq$ $9 \Lambda /(14+2 \sqrt{85})$. For $\gamma>\Lambda / 5$, the instability is caused by the eigenvalue (41) becoming negative, which occurs when $\gamma=\gamma_{\mathrm{cr}}^{2}$, where

$$
\gamma_{\text {cr }}^{2}=\sqrt{\Lambda^{2}-4 \Lambda C}
$$


this value is meaningful only when $C \leq \Lambda / 4$.

Furthermore, if we include terms up to $\mathcal{O}\left(C^{2}\right)$, we obtain

$$
\Omega=\Lambda^{2}-\gamma^{2}-4 \Lambda C+4 C^{2}+\mathcal{O}\left(C^{3}\right)
$$

as the eigenvalue of an onsite discrete dark soliton. Using this expression, we find the critical value of $\gamma$ indicating the onset of instability to be

$$
\gamma_{\mathrm{cr}}^{1}=-\frac{2}{5} \Lambda-\frac{8}{5} C+\frac{1}{5} \sqrt{9 \Lambda^{2}-28 \Lambda C+4 C^{2}},
$$

for $\gamma<0.2 \Lambda$ and

$$
\gamma_{\mathrm{cr}}^{2}=\sqrt{\Lambda^{2}-4 \Lambda C+4 C^{2}}
$$

for $\gamma \geq 0.2 \Lambda$.

\section{Intersite modes}

Intersite discrete dark solitons are given, with errors of $\mathcal{O}\left(C^{2}\right)$, by

$$
u_{n}= \begin{cases}-\sqrt{\Lambda+\gamma}, & n=-2,-3, \ldots \\ -\sqrt{\Lambda+\gamma}+C / \sqrt{\Lambda+\gamma}, & n=-1 \\ \sqrt{\Lambda+\gamma}-C / \sqrt{\Lambda+\gamma}, & n=0 \\ \sqrt{\Lambda+\gamma}, & n=1,2, \ldots\end{cases}
$$

Starting from Eq. (34), we then find

$$
\mathcal{L}_{+}(0) \mathcal{L}_{-}(0)=4(\Lambda+\gamma) \gamma
$$

for all $n$, from which we deduce that there is a single leading-order eigenvalue, given by $\Omega^{(0)}=4(\Lambda+\gamma) \gamma$, with infinite multiplicity. This eigenvalue then expands to form the continuous spectrum for nonzero $C$.

Because a localized structure must have an eigenvalue, we infer that an eigenvalue will bifurcate from the lower edge of the continuous spectrum. This bifurcating eigenvalue may be calculated from Eq. (35). Because

$$
\mathcal{L}_{+}^{(0)}(0) \mathcal{L}_{-}^{(0)}(0)-\Omega^{(0)}=0
$$

for all $n$, the solvability condition for Eq. (35) requires $f_{n}=0$ for all $n$. A simple calculation then yields

$f_{n}= \begin{cases}{\left[4 \Lambda+16 \gamma+(2 \Lambda+4 \gamma) \Delta_{2}+\Omega^{(1)}\right] \eta_{n}^{(0)},} & n=-1,0 \\ {\left[(2 \Lambda+4 \gamma) \Delta_{2}+\Omega^{(1)}\right] \eta_{n}^{(0)},} & n \neq-1,0\end{cases}$

Taking $\eta_{n}^{(0)}=0$ for $n \neq-1,0$ leaves the two nontrivial equations

$$
\begin{aligned}
& \left(8 \gamma+\Omega^{(1)}\right) \eta_{-1}^{(0)}+(2 \Lambda+4 \gamma) \eta_{0}^{(0)}=0 \\
& \left(8 \gamma+\Omega^{(1)}\right) \eta_{0}^{(0)}+(2 \Lambda+4 \gamma) \eta_{-1}^{(0)}=0
\end{aligned}
$$

from which we see that $\eta_{-1}^{(0)}= \pm \eta_{0}^{(0)}$. Thus we obtain two possibilities for the $\mathcal{O}(C)$ contribution to the eigenvalue, given by

$$
\Omega_{1}^{(1)}=-(12 \gamma+2 \Lambda), \quad \Omega_{2}^{(1)}=2 \Lambda-4 \gamma .
$$

Hence the eigenvalues bifurcating from the lower edge of the continuous spectrum are given by

$$
\Omega_{1}=4(\Lambda+\gamma) \gamma-(12 \gamma+2 \Lambda) C+\mathcal{O}\left(C^{2}\right)
$$

and

$$
\Omega_{2}=4(\Lambda+\gamma) \gamma+(2 \Lambda-4 \gamma) C+\mathcal{O}\left(C^{2}\right)
$$

$$
\gamma<0.5 \Lambda
$$
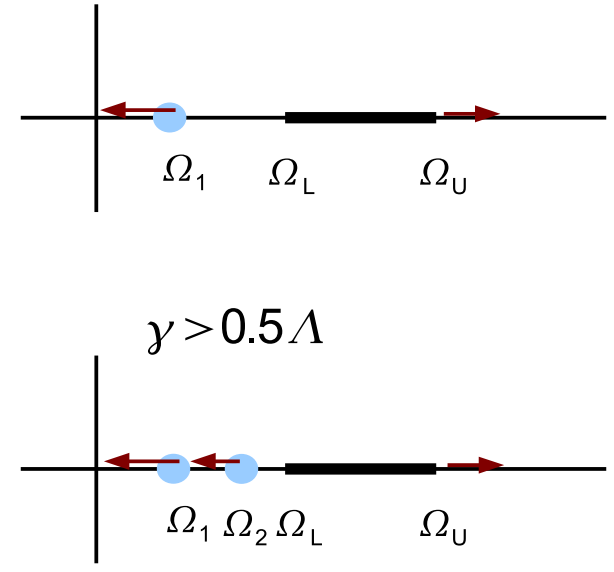

FIG. 8: As Fig. 1 but for a stable intersite dark soliton.

A simple analysis shows that $\Omega_{2}<\Omega_{L}$ only when $\gamma>$ $\Lambda / 2$. The sketch in Fig. 8 then illustrates that instability is caused by $\Omega_{1}$ becoming negative. This consideration gives the critical $\gamma$ as a function of the coupling constant $C$ to be

$$
\gamma_{\mathrm{cr}}=-\frac{1}{2} \Lambda+\frac{3}{2} C+\frac{1}{2} \sqrt{\Lambda^{2}-4 \Lambda C+9 C^{2}} .
$$

When there are two eigenvalues $\left(\Omega_{1}\right.$ and $\left.\Omega_{2}\right), \Omega_{2}$ decreases more slowly than $\Omega_{1}$, in such a way that for $\gamma>\Lambda / 2$ the instability is still caused by $\Omega_{1}$ becoming negative.

\section{B. Comparison with numerical computations}

\section{Onsite dark solitons}

We now compare our analytical results with corresponding numerical calculations. As for bright solitons, for illustrative purposes we set $\Lambda=1$.

We start by checking the validity of our analytical approximation for the critical eigenvalue associated with the phase mode. As explained above, the change in the position of the eigenvalues relative to the continuous spectrum at $C=0$ occurs at $\gamma=1 / 5$. Therefore we consider the two values $\gamma=0.1$ and $\gamma=0.6$, representing both cases. Figure 9 depicts a comparison between our analytical result Eq. (41) and the numerical computations, from which we conclude that the prediction is 


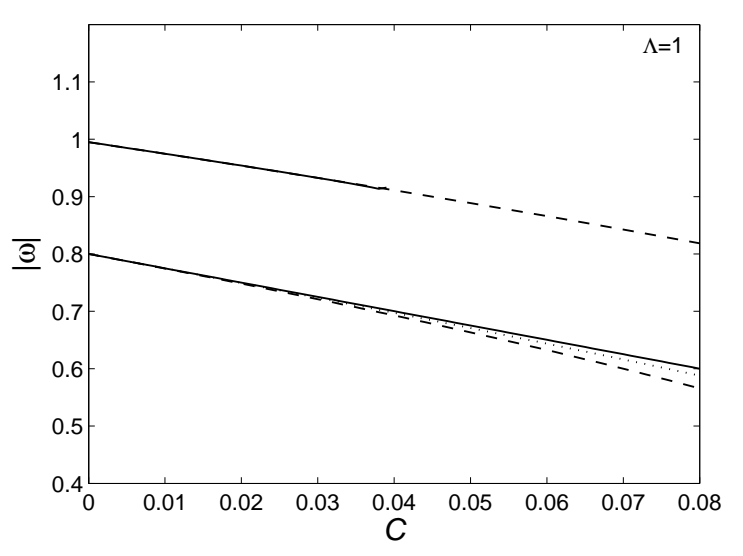

FIG. 9: Comparisons between the critical eigenvalue for onsite dark solitons obtained numerically (solid lines) and analytically using Eq. (41) (dashed lines) for $\gamma=0.1$ (upper curves) and $\gamma=0.6$ (lower curves). An approximation that explicitly includes the next term in expansion Eq. (44) is also plotted (dotted lines).

quite accurate for small $C$. The accuracy can be improved if one includes further orders in the perturbative expansion Eq. (44), and this improvement is shown in the same figure by the dotted line.

The eigenvalue structure of onsite dark solitons is depicted in Fig. 10 left and right panels refer respectively to conditions just before and just after a collision resulting in an instability.

As sketched in Fig. 7 , for $\gamma<1 / 5$ the instability is caused by a collision between the eigenvalue and one edge of the continuous spectrum. On the other hand, when $\gamma \geq 1 / 5$ the instability is caused by a collision between the eigenvalue and its twin at the origin (see the bottom panels of Fig. 10).

We now proceed to evaluate the (in)stability region of this solution in $(C, \gamma)$ space. Shown in Fig. 11 is again the maximum of the imaginary part of the eigenvalue, together with our approximation to the (in)stability boundary. The white dashed line represents Eq. (43), corresponding to the instability caused by the collision with the continuous spectrum. Equation (42) is represented by the white dash-dotted line, which corresponds to the other instability mechanism. In addition, pink dashed and dash-dotted lines show, respectively, Eq. (46) and Eq. (45), where a better analytical approximation is obtained.

An important observation from the figure is that there is an interval of values of $\gamma$ in which the onsite dark soliton is always stable, for any value of the coupling constant $C$. This indicates that a parametric driving can fully suppress the oscillatory instability reported for the first time in 21].

\section{Intersite dark solitons}

Now we examine intersite dark solitons.

Firstly, Fig. 12 shows the analytical prediction for the critical eigenvalue, given by Eq. (51), compared to numerical results. We see that the approximation is excellent for small $C$ and its range of validity is wider for larger values of $\gamma$. The eigenvalue structure of this configuration is shown in Fig. 13 for the two values $\gamma=0.1,0.8$. The mechanism of instability explained in the section above can be seen clearly in the top panels of Fig. 13 .

It is interesting to note that a parametric driving can also fully suppress the oscillatory instability of an intersite dark soliton. As shown in the bottom panels of Fig. 13, there are values of the parameter $\gamma$ for which no instability-inducing collision ever occurs. The (in)stability region of this configuration is summarized in Fig. 14, where we see that for any $C$ and $\gamma>0.3$ an intersite dark soliton is always stable. Our analytical prediction for the onset of instability is given by the dashed line in that figure. We observe that for relatively small $C$, the prediction of Eq. (51) is reasonably close to the numerical results.

\section{DISCUSSION}

In the sections above we discussed the existence and the stability of localized modes through our reduced DNLS equation (5). In this section, we confirm the relevance of our findings through solving numerically the original time-dependent equation (11). We use a Runge-Kutta integration method, with the initial condition $\varphi_{n}=2 \epsilon u_{n}$ and $\dot{\varphi}_{n}=0$, where $u_{n}$ is the static solution of the DNLS (5) and $\epsilon$ is the small parameter of Sec. I. Throughout this section, we use the illustrative value $\epsilon=0.2$.

Shown in the left and right panels of Fig. [15] are the numerical evolution of a stable and unstable onsite bright soliton, respectively. From the right panel of the figure, we note that a parametric driving seems to destroy an unstable soliton. This observation is similar to the corresponding observation for the dynamics of an unstable soliton in the DNLS equation (5) reported in Ref. 25.

In Fig. 16 we present the numerical evolution of intersite bright solitons for the same parameter values as those in Fig. [5] corresponding to each of the instability scenarios. From the panels in this figure, we see that the typical dynamics of the instability is in the form of soliton destruction or discharge of a traveling breather.

We have also examined the dynamics of onsite dark solitons in the Klein-Gordon system (10). Shown in Fig. 17 is the numerical evolution of a solution with the eigenvalue structure illustrated in Fig. 10. The instability of an unstable onsite dark soliton typically manifests itself in the form of oscillations in the location of the soliton center about its initial position (top right panel) 


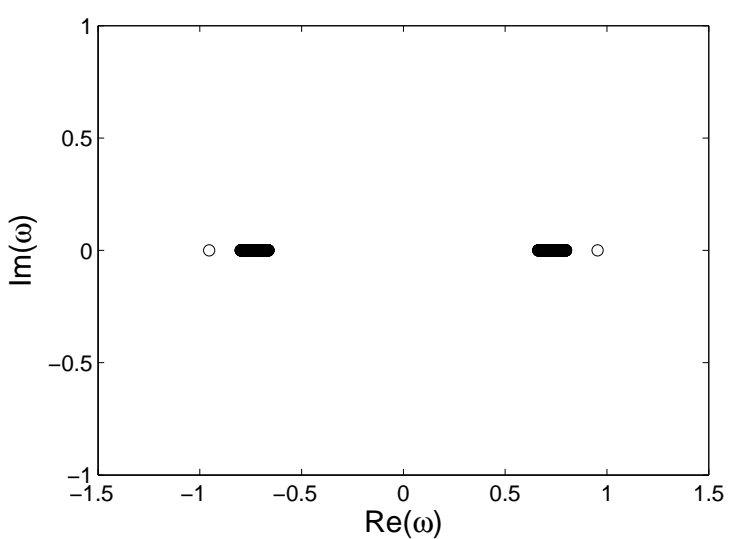

(a) $\gamma=0.1, C=0.02$

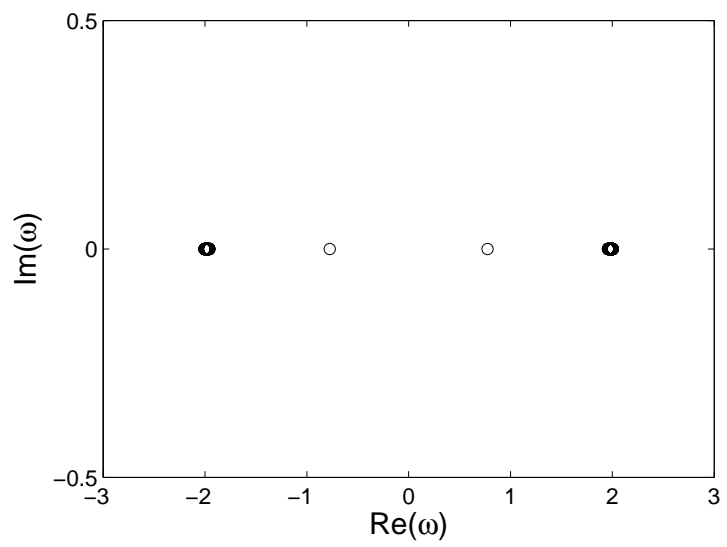

(c) $\gamma=0.6, C=0.01$

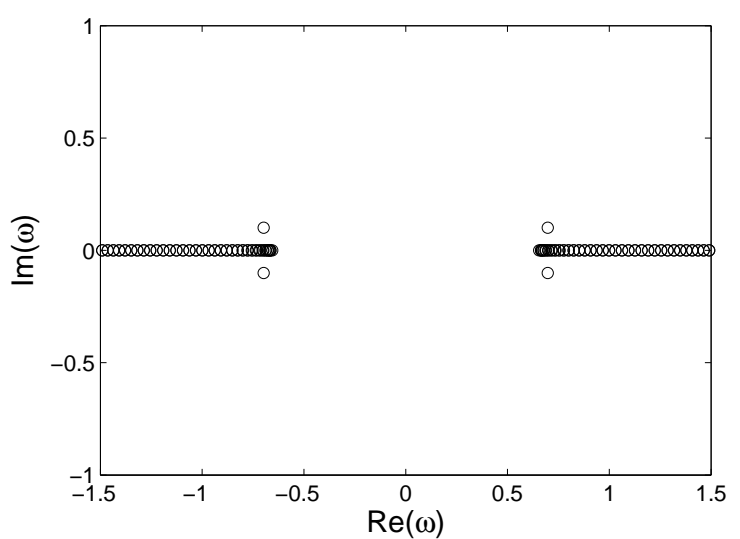

(b) $\gamma=0.1, C=0.2$

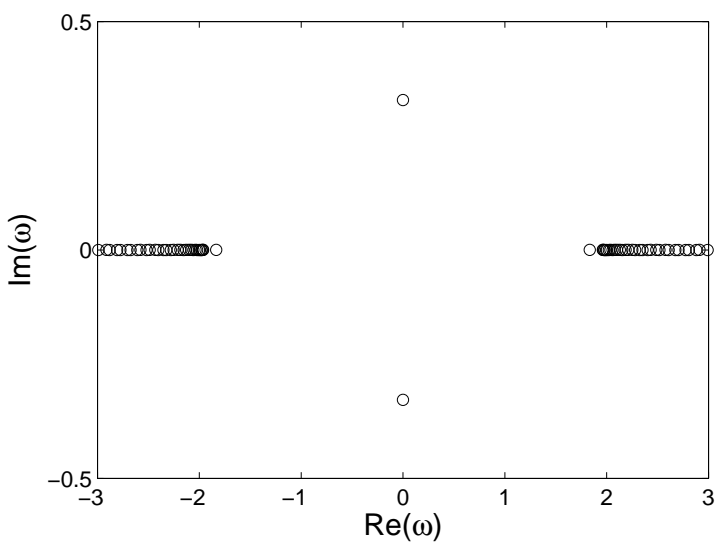

(d) $\gamma=0.6, C=1$

FIG. 10: The eigenvalue structure of on-site dark solitons for several values of $\gamma$ and $C$, as indicated in the caption of each panel.

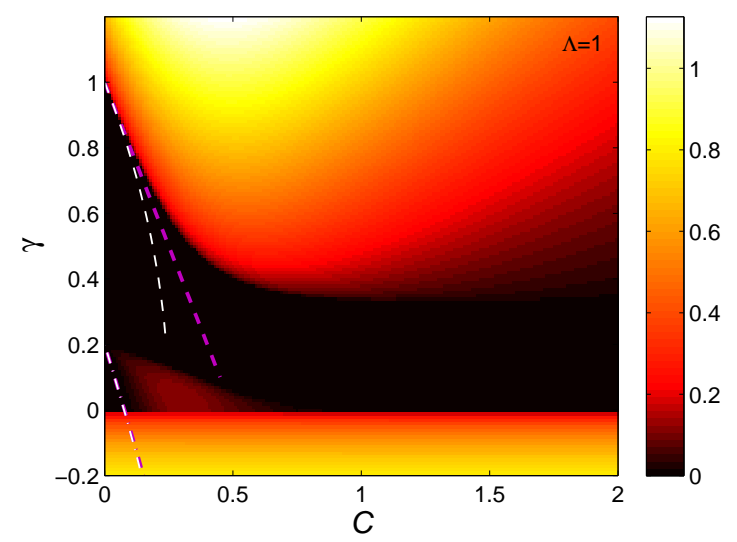

FIG. 11: (Colour online) The (in)stability region of onsite dark solitons in the two-parameter $(C, \gamma)$ space. The white and pink dashed lines respectively give the analytical approximations Eq. (43) and (46). White and pink dash-dotted lines show Eqs. (42) and (45); note that these curves are indistinguishable in this plot.

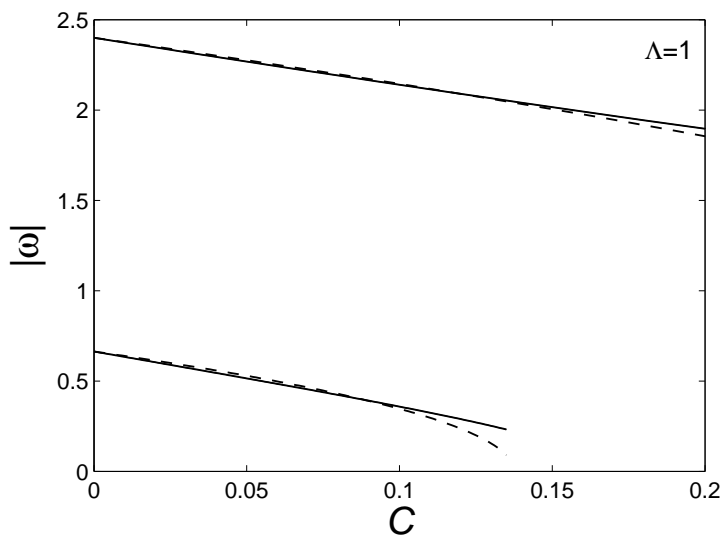

FIG. 12: Comparisons between the critical eigenvalue for intersite dark solitons obtained numerically (solid lines) and analytically (dashed lines) using Eq. (51), for two values of $\gamma$. The upper curves correspond to $\gamma=0.8$, and the lower ones to $\gamma=0.1$. 


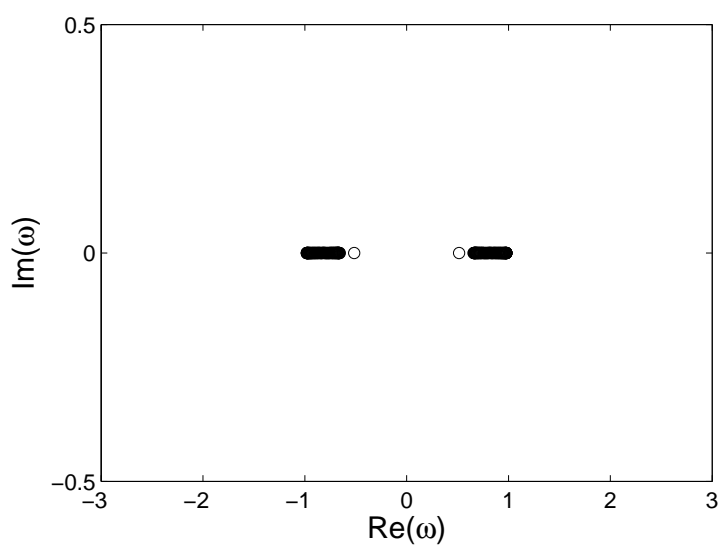

(a) $\gamma=0.1, C=0.05$

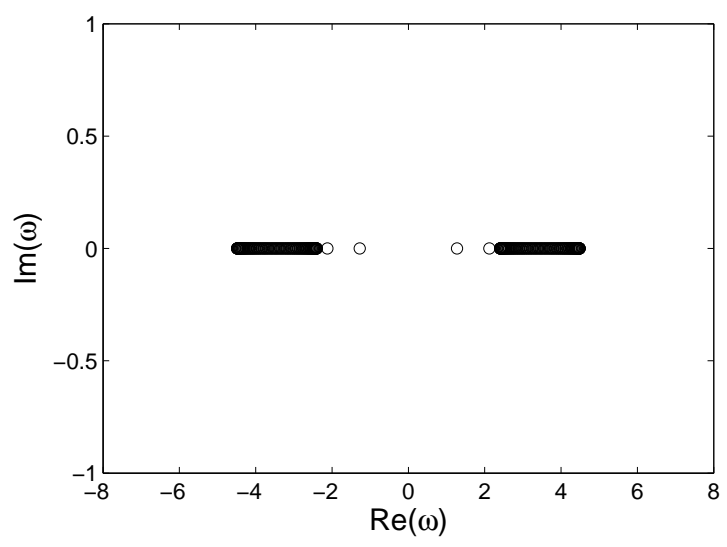

(c) $\gamma=0.8, C=0.5$

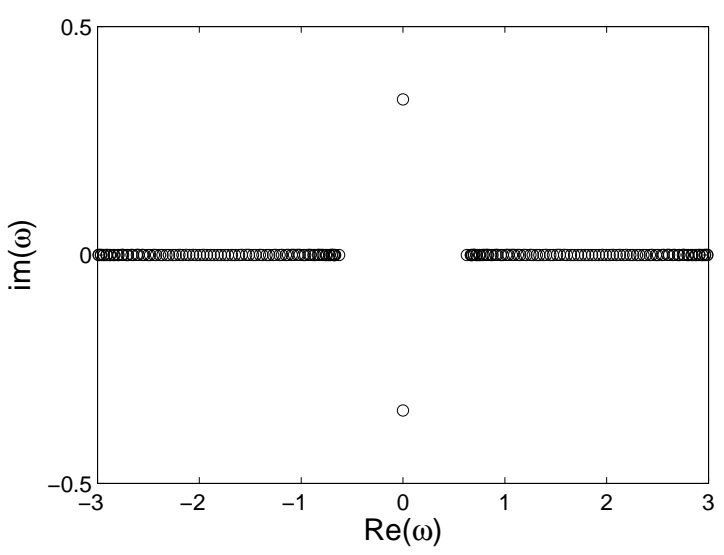

(b) $\gamma=0.1, C=0.5$

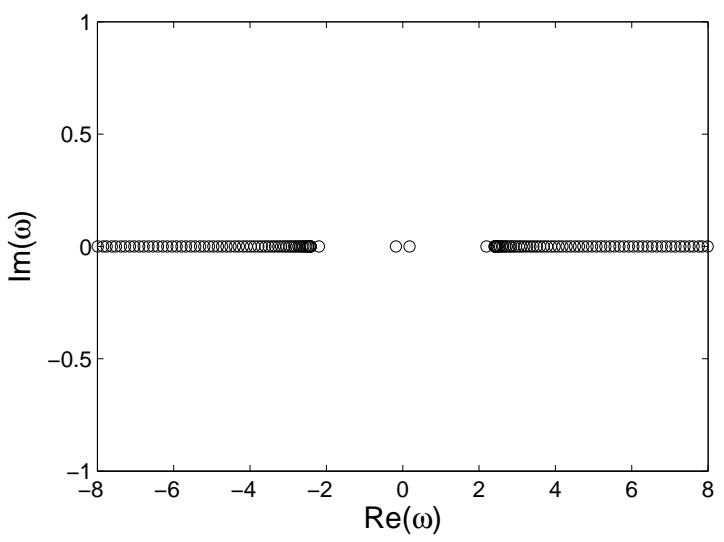

(d) $\gamma=0.8, C=2$

FIG. 13: The eigenvalue structure of intersite dark solitons with parameter values as indicated in the caption for each panel.

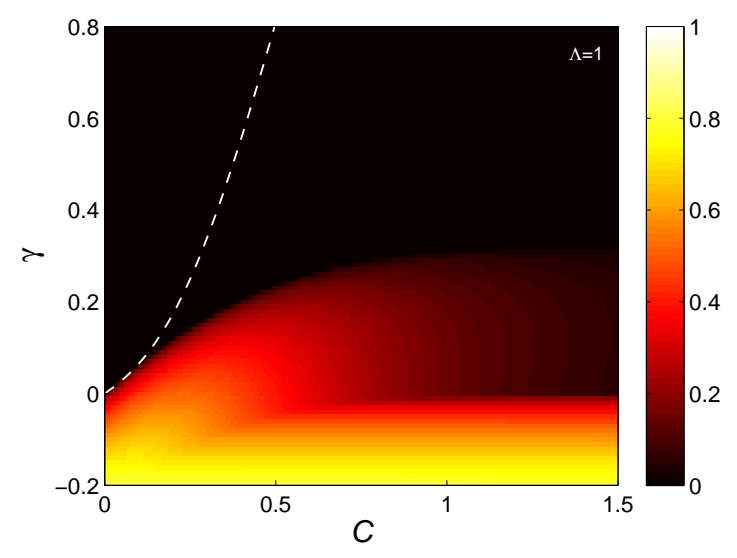

FIG. 14: (Colour online) As Fig. 11] but for an intersite dark soliton. The white dashed line is our analytical approximation Eq. (53).

or oscillations in the width of the soliton (bottom right panel).

Finally, we illustrate the dynamical behavior of an un- stable intersite dark soliton in Fig. 18, from which we see that the instability makes the soliton travel. This dynamics is similar to that reported in Ref. 20.

\section{CONCLUSION}

In this paper, we have considered a parametrically driven Klein-Gordon system describing nanoelectromechanical systems. Using a multiscale expansion method we have reduced the system to a parametrically driven discrete nonlinear Schrödinger equation. Analytical and numerical calculations have been performed to determine the existence and stability of fundamental bright and dark discrete solitons in the Klein-Gordon system through use of the Schrödinger equation. We have shown that the presence of a parametric driving can destabilize an onsite bright soliton. On the other hand, a parametric driving has also been shown to stabilize intersite bright and dark discrete solitons. We even found an interval in $\gamma$ for which a discrete dark soliton is stable for any value of the coupling constant, i.e. a parametric driving can suppress oscillatory instabilities. Stability windows 

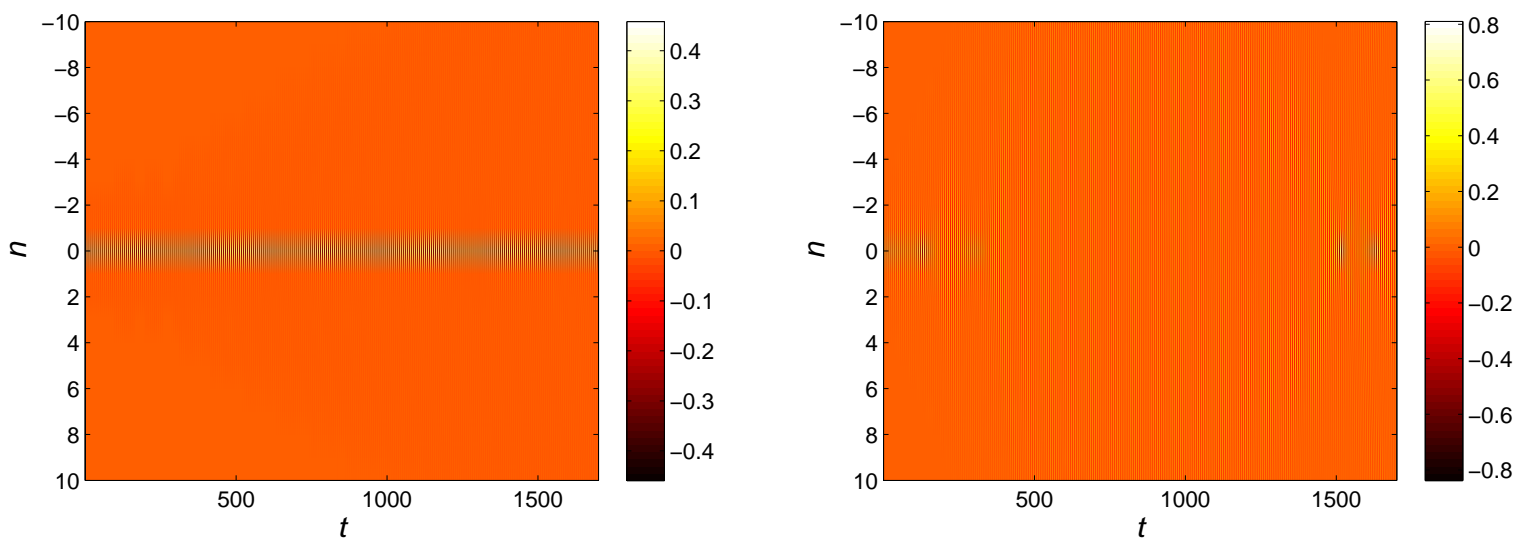

FIG. 15: (Colour online) The spatio-temporal evolution of an onsite bright soliton governed by the original time-dependent parametrically driven Klein-Gordon system (1), with $\epsilon=0.2$ and $\gamma=0.1$. The left and right panels show a stable and unstable soliton, at $C=0.1$ and $C=1$, respectively.

for all the fundamental solitons have been presented and approximations using perturbation theory have been derived to accompany the numerical results. Numerical integrations of the original Klein-Gordon system have demonstrated that our analytical and numerical investigations of the discrete nonlinear Schrödinger equation provide a useful guide to behavior in the original system.
[1] M. D. Ventra, S. Evoy, and J. R. Heflin, Introduction to nanoscale science and technology (Springer, 2004).

[2] A. Cleland, Foundation of Nanomechanics (Springer, New York, 2002).

[3] M. L. Roukes, Phys. World 14, February 2001.

[4] A. N. Cleland and M. L. Roukes, Nature 392, 160 (1998).

[5] I. Katz, R. Lifshitz, A. Retzker, and R. Straub, New J. Phys. 10, 125023 (2008).

[6] E. Buks and M. L. Roukes, J. Microelectromech. Syst. 11, 802 (2002).

[7] J. Wiersig, S. Flach, and K.-H. Ahn, Appl. Phys. Lett. 93, 222110 (2008).

[8] M. Sato, B. E. Hubbard, and A. J. Sievers, Rev. Mod. Phys. 78, 137-157 (2006).

[9] M. Sato and A. J. Sievers, Phys. Rev. Lett. 98, 214101 (2007).

[10] M. Sato, S. Yasui, M. Kimura, and A. J. Sievers, Europhys. Lett. 80, 30002 (2007).

[11] E. Kenig, B. A. Malomed, M. C. Cross, R. Lifshitz, arXiv:0904.1355.

[12] R. Lifshitz and M. C. Cross, Phys. Rev. B 67, 134302 (2003).

[13] S. C. Masmanidis, R. B. Karabalin, I. De Vlaminck, G. Borghs, M. R. Freeman, and M. L. Roukes, Science 317, 780 (2007).

[14] Yu. S. Kivshar, Phys. Lett. A 173, 172-178 (1993).

[15] M. Remoissenet, Phys. Rev. B 33, 2386 (1986).

[16] Yu. A. Kosevich and G. Corso, Physica D 170, 1-12 (2002).

[17] D. Hennig and G. Tsironis, Phys. Rep. 307, 333-432 (1999)

[18] G. L. Alfimov, V. A. Brazhnyi, and V. V. Konotop, Phys. D 194, 127-150 (2004).
[19] D. E. Pelinovsky, P. G. Kevrekidis, and D. J. Frantzeskakis, Physica. D 212, 1-19 (2005).

[20] E. P. Fitrakis, P. G. Kevrekidis, H. Susanto, and D. J. Frantzeskakis, Phys. Rev. E 75, 066608-12 (2007).

[21] M. Johansson and Yu. S. Kivshar, Phys. Rev. Lett. 82, 85 (1999)

[22] Yu. S. Kivshar, W. Królikowski, and O.A. Chubykalo, Phys. Rev. E 50, 5020 (1994).

[23] H. Susanto and M. Johansson, Phys. Rev. E 72, 016605 (2005).

[24] D. E. Pelinovsky and P. G. Kevrekidis, J. Phys. A. 41, 185206 (2008).

[25] H. Susanto, Q. E. Hoq, and P. G. Kevrekidis, Phys. Rev. E 74, 067601-4 (2006).

[26] I. V. Barashenkov, Phys. Rev. Lett. 77, 1193 (1996).

[27] I. V. Barashenkov, E. V. Zemlyanaya, and M. Bär, Phys. Rev. E 64, 016603 (2001).

[28] I. V. Barashenkov, S. R. Woodford, and E. V. Zemlyanaya, Phys. Rev. Lett. 90, 054103 (2003).

[29] I. V. Barashenkov, S. Cross, and B. A. Malomed, Phys. Rev. E 68, 056605 (2003).

[30] I. V. Barashenkov and S. R. Woodford, Phys. Rev. E 71, 026613 (2005).

[31] I. V. Barashenkov, S. R. Woodford, and E. V. Zemlyanaya, Phys. Rev. E 75, 026604 (2007).

[32] I. V. Barashenkov and S. R. Woodford, Phys. Rev. E 75, 026605 (2007).

[33] V. M. Kaurov and A. B. Kuklov, Phys. Rev. A 71, 011601 (2005)

[34] V. M. Kaurov and A. B. Kuklov, Phys. Rev. A 73, 013627 (2006). 


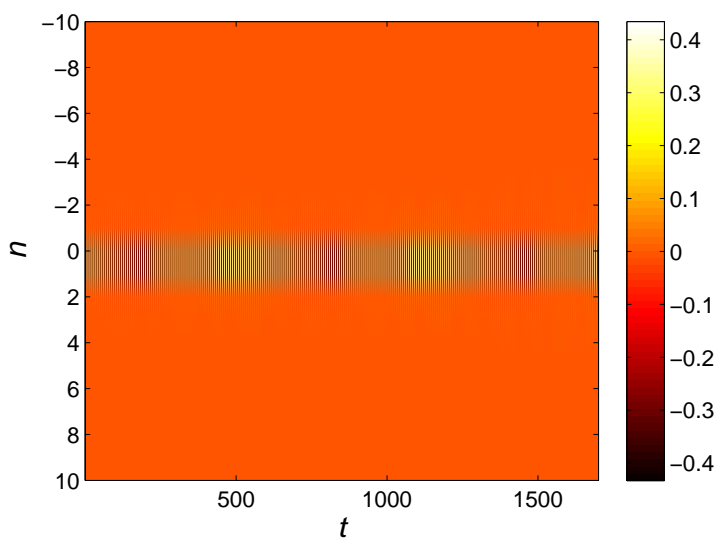

(a) $\gamma=0.1, C=0.05$

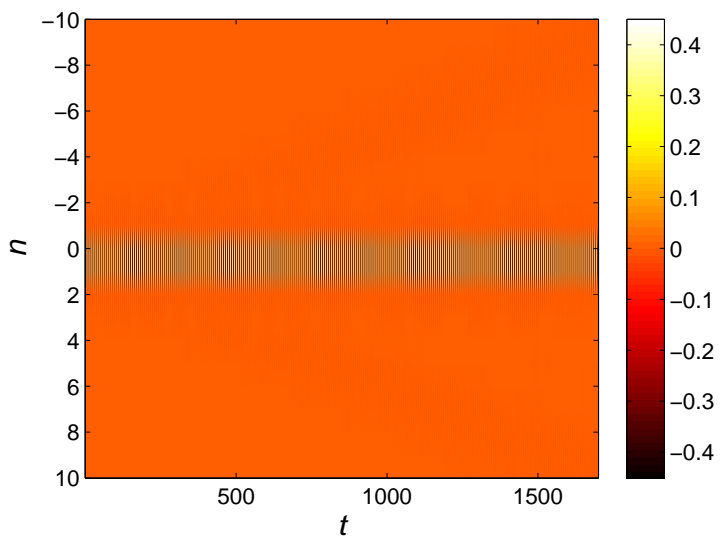

(c) $\gamma=0.18, C=0.05$

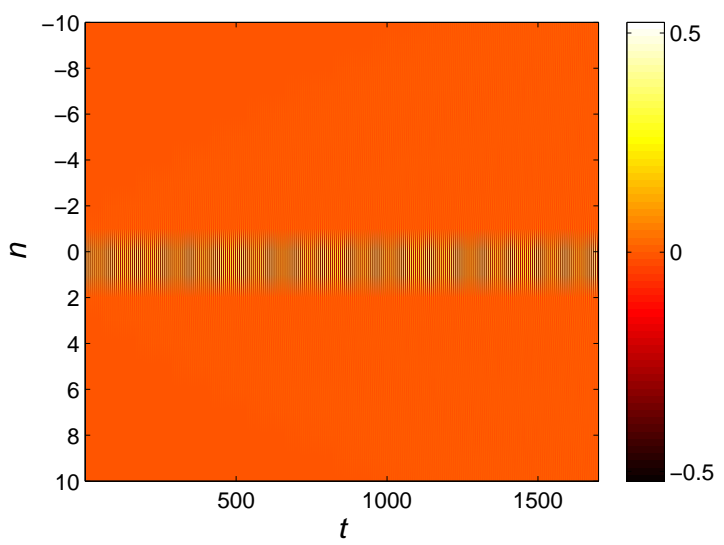

(e) $\gamma=0.5, C=0.05$

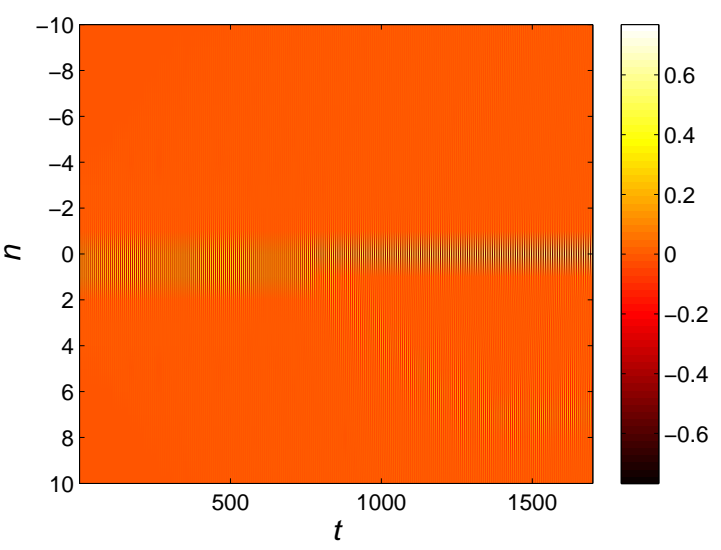

(b) $\gamma=0.1, C=0.3$

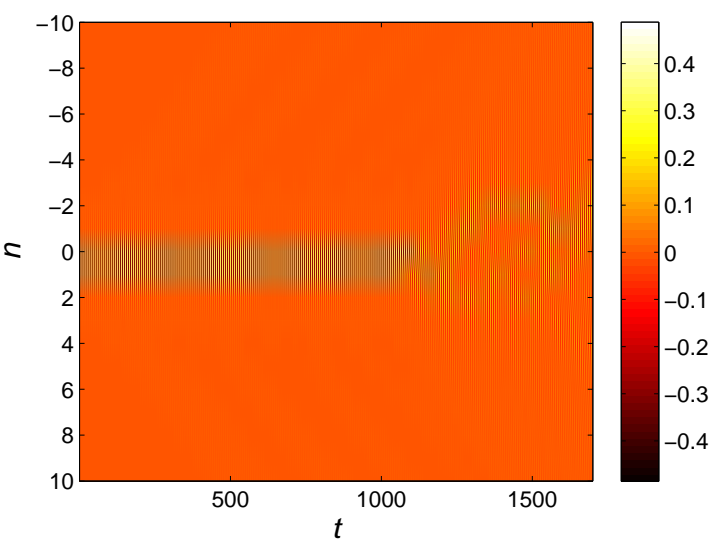

(d) $\gamma=0.18, C=0.18$

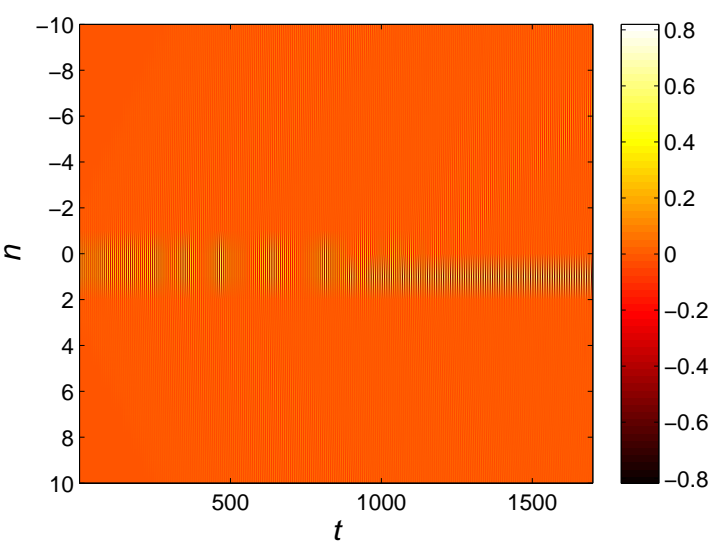

(f) $\gamma=0.5, C=0.2$

FIG. 16: (Colour online) As Fig. 15] but for an intersite bright soliton, with parameter values as indicated in the caption for each panel. The initial profile in each panel corresponds to the same parameters as in Fig. 5 . 


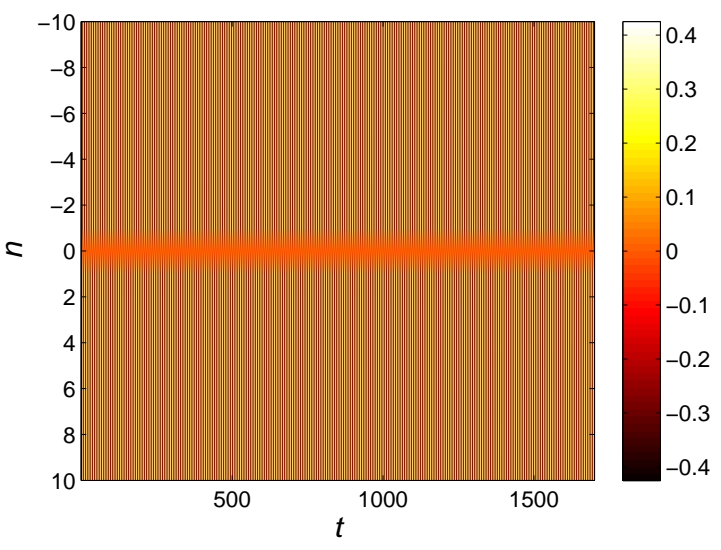

(a) $\gamma=0.1, C=0.02$

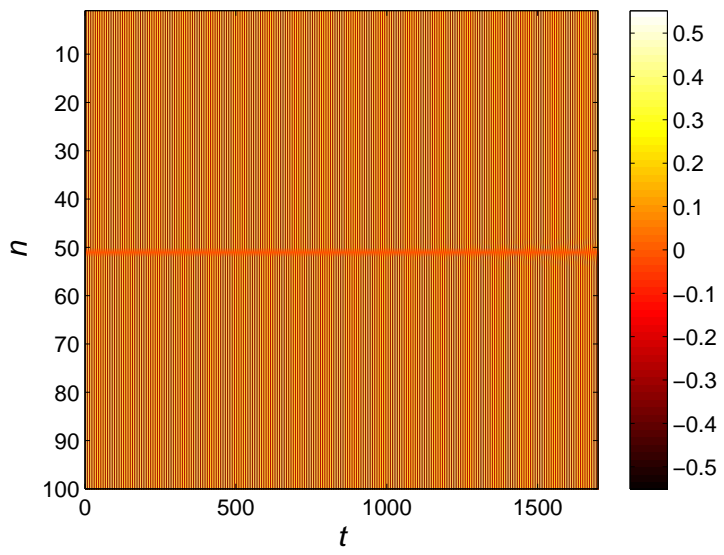

(c) $\gamma=0.6, C=0.01$

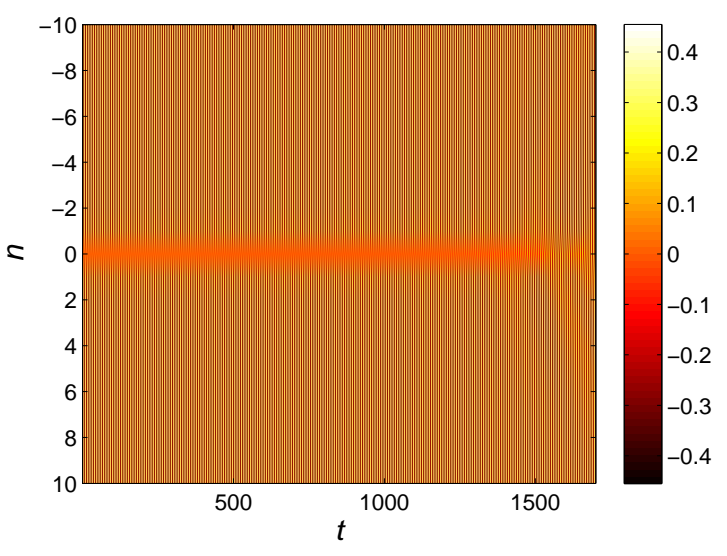

(b) $\gamma=0.1, C=0.2$

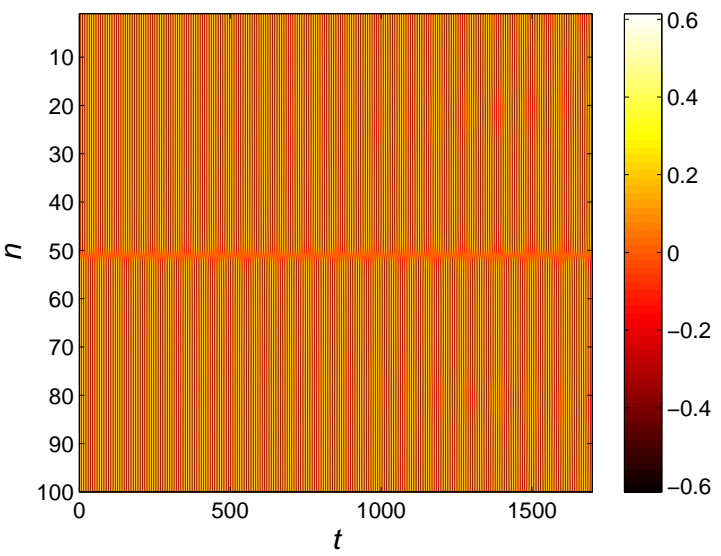

(d) $\gamma=0.6, C=1$

FIG. 17: (Colour online) As Fig. 15] but for on-site dark solitons. The parameter values are as in Fig. 10
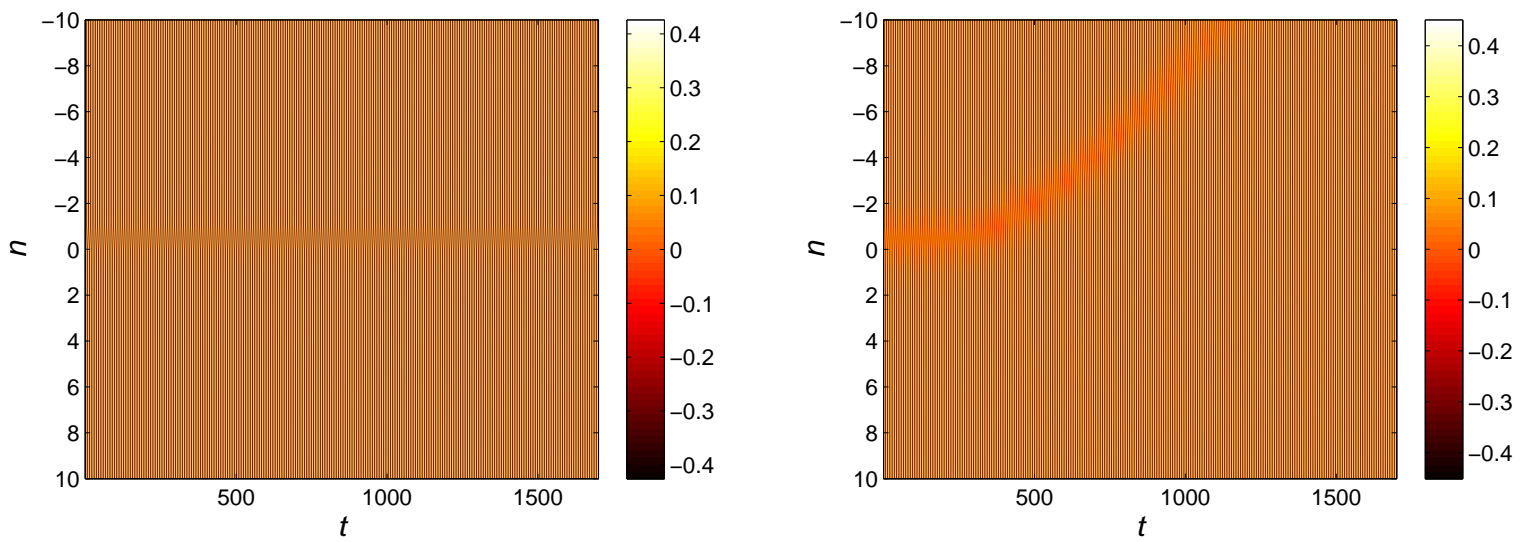

FIG. 18: (Colour online) As Fig. 15, but for an intersite dark soliton with $\gamma=0.1$. The left panel shows the evolution of a stable dark soliton with $C=0.05$, while the right panel shows the evolution of an unstable dark soliton with $C=0.5$. 